\title{
The Application of Parallel Corpus in the Teaching of English Translation of Traditional Chinese Medicine
}

\author{
Cheng Liu ${ }^{1, a}$ and Xiaofang Wang ${ }^{2, b^{*}}$ \\ ${ }^{1}$ School of Humanities, Jiangxi University of TCM, No. 1688 of Meiling Road, Wanli District, \\ Nanchang City, Jiangxi Province, China \\ ${ }^{2}$ School of Humanities, Jiangxi University of TCM, No. 1688 of Meiling Road, Wanli District, \\ Nanchang City, Jiangxi Province, China \\ ajxjzlc@163.com, b920711622@qq.com \\ * please mark the corresponding author with an asterisk
}

Keywords: Basic terms of TCM; English synonym corpus; Translation teaching

\begin{abstract}
With the development of the research on corpus, the corpus of the network resources has become a convenient and economic language teaching resource. The self-made traditional Chinese medicine(TCM)parallel corpus was used for TCM translation teaching. By means of data presentation, retrieval query techniques and methods, systematic and efficient translation teaching, the organic combination of traditional translation teaching and corpus technology, the TCM parallel corpus can more effectively promote the quality of the translation of TCM teaching.
\end{abstract}

\section{Introduction}

With the continuous development of computer application technology, the research of corpus based on linguistics and computer science has achieved fruitful results. Corpus linguistics is based on the real language data as the object of study, from a macro point of view of a large number of linguistic facts to analyze, and to sum up the rules of language use, to reflect new research ideas. Many countries have established different scales and different types of corpuses. The corpus processing degree is deeper and deeper, and the application scope is more and more widely. Corpus as the development and utilization of a new type of English teaching resources has great research space, how to use corpus resources more effectively will become a new era and the majority of English teaching researchers are keen to pay attention to the problem. This paper mainly introduces the application of the self made TCM terminology parallel corpus in the teaching of TCM translation teaching.

\section{A Brief Introduction to the TCM Terminology Parallel Corpus}

Compared with dictionary and monolingual corpora, the parallel corpus has obvious advantages. The parallel corpus is bilingual, plenty of real context, and strong timeliness.

Researchers with the help of a large number of data, can compare content from the aspects of semantics, syntax and pragmatics. Researchers can also analyze the characteristics of the language in depth, and reveal the cultural differences between the original and the translation and so on. This study uses the corpus technology and establishes a self made TCM terminology parallel corpus which relates to the basic theory of Chinese medicine, diagnostics, formulas of Chinese medicine, Chinese medicine terminology and text translation.

\section{The Main Content of the Study}

This project utilized self-made TCM terminology bilingual corpus, Birmingham English Chinese parallel corpus and British National Corpus, and made qualitative analysis, quantitative analysis, and macro summary and micro analysis of the language text to promote the research of translation teaching. 
Promotion of Data-driven Learning. In translation teaching, teachers first teach students to use paraConc bilingual concordance tool to retrieve corpus by themselves. In the dynamic environment, students can master and observe the collocation of words, and then summarize and induce the expression of linguistic meaning, pragmatic features and grammatical rules, etc. Teachers apply the typical errors and the good work in students' practice to the teaching process. Teachers typed all the students' answers into the corpus, and could directly retrieve a definite answer of any question. So teachers can find the widespread problems and the main cause of the error.

Probability Analysis of Text Equivalence. With the help of the parallel corpus, users can do the equivalent probability analysis of professional terminology in the use of classical works of TCM and the modern literary style. Users can also do the equivalent probability analysis of different professional terms in the same style, and summarize the rules of translating this type of text.

In the process of translation teaching, the selected words are translated according to the frequency rate, and are marked their meanings both in TCM English and common English, which is beneficial for teachers and students to do comparison and comprehend the professional meaning and usage of the words. In translation practice, we should follow the principle of going from easy to difficult and from words to phrases and sentences. With the help of the corpus we obtain the professional sentences of TCM, which is good for students to enrich the translated words, feel the translation context, and find more appropriate and more idiomatic translations, improve students' sense of language and professional translation as well as the translation quality.

A Comparative Study of Several Translation Versions. The self-made TCM terminology parallel corpus provides a more comprehensive synonym translation words or phrases, and multiple translations of a word. This corpus puts several versions of TCM terminology, which can help users to decide which one is the best translation. It can also help users to analyze the general characteristics of translation texts, to explore the general rules of English translation of TCM. It can help users to apply domestication and foreignization strategies appropriately as well. This parallel corpus can inspire students to create more excellent works of TCM.

\section{The Application of the Parallel Corpus of Chinese Medicine in the Teaching of English Translation of Traditional Chinese Medicine}

In order to improve the teaching quality and efficiency, the research group designed TCM basic terminology parallel corpus from three aspects based on the 32 teaching hours of TCM English translation.

Corpus Presentation. An important part of translation teaching is to cultivate learners' cognitive ability of the differences between source language and target language, and a large number of parallel texts have an irreplaceable role in training learners' cognitive ability.[6] Teachers selected and printed 10 sampled TCM English text for students (Each piece has about 300 words), then distributed to the students a week ahead of time. In the first week of class hour, teachers will explain and analyze the macro structure of the text and language style. Students will form of an intuitive understanding of the initial understanding of the translation of TCM in the specific translation of TCM materials. At the beginning of class time in the second week, teachers will present and explain language phenomenon of sub themes of the corpus, such as the vocabulary of basic theory of TCM, TCM diagnosis vocabulary, vocabulary acupuncture, Chinese medicine and prescription lexicology. Through self-made bilingual corpus, the author can easily extract what he needs from the corpus, which not only reduce the burden on teachers' compilation, but also get plenty of high quality teaching materials, and reach persuasive teaching effect.

Highfrequency Chunks. In the teaching of TCM English translation, time for teachng of vocabulary is limited, and the emphasis of the teaching should be on the most common basic words and expressions. Through the wordlist function of the software Antconc, Chinese words and English words of high frequency or high frequency vocabulary chunks are generated. Lexical chunks refer to a muli word sequence with high frequency and complete meaning ${ }^{[7]}$. Proficiency in a language, to a large extent, is to master the way of expression, the language of the number of chunks. Through the N-Gram function 
of Antconc, it can be easily extracted from TCM words and English Chinese medicine words. In teaching process, required chunks were extracted, and the frequency was determined, then through artifiial screening, chunks with incomplete grammatical structure or meaning expression will be excluded finaly, eventually about 800 Chinese and English high frequency chunks will be attained. Then the corresponding English and Chinese expressions are retrieved respectively, and then the teaching materials are sent to the students.

Search Query. Comared with large proprietary parallel corpus, the TCM parallel corpus is more targeted. For translation teaching and students' autonomous learning, the most simple and easy use of parallel corpus is the retrieval of the equivalent vocabulary [4]. English translation of TCM course employs project teaching. In 32 teaching hours, students in each group have to complete the translation of the five pieces of TCM materials and simulated conversation tasks, and the conversation tasks are to be carried out after class. Teachers will copy bilingual database of TCM and Demo Paraconc software to the students, in order to facilitate their search for their own inquiries and solve the problems encountered in the translation of Chinese medical terminology. Problems they cannot solve wii be given to the teacher. Many students reflect this way can greatly improve their translation quality and efficiency.

\section{Conclusion}

In this paper, the technique of corpus is applied into translation teaching, which can be done systematically and efficiently through the corpus representation, high frequency chunks and search query and other methods. And appropriate control is also very important in the teaching process and we also need reasonable groupng, computer technology training and analysis of students' translation works. This teaching application needs to be improved further. It is still difficult for students to fully digest the subject material and high frequency chunks and other materials surviving. In addition, while querying and understanding the different translation versions, students have difficulty in lanuage ability, analysis ability and retrieval technology ${ }^{[8]}$. So this new translation teaching mode cannot completey deny the traditional translation teaching. Teachrs should better combine two kinds of teaching philosohy in teaching and play to the strengths of the two teaching methods in order to improve the quality of the translation teaching of TCM more effectively.

\section{Acknowledgements}

Study of English translation of basic terms of Traditional Chinese Medicine based on corpus (YY162002); Special Social Science Planning Project of Foreign Language Teaching and Research of Universities and Colleges in Jiangxi Province (No.15WX201); Key project of Teaching Reform of Jiangxi University of traditional Chinese Medicine in 2016 (No.2016jzzd-1).

\section{References}

[1]Yang Mei, Bai Nan. Status survey of domestic corpus translation studies [J]. China translation, 2010, (6): 46-50.

[2] Qin Hongwu, Tu Kefei. Application of corpus used in Translation Teaching: theoretical basis and implementation principle [J]. Chinese translation, 2007 (5):49-52.

[3] Wang Kefei. The use of bilingual parallel corpus in translation teaching [J]. Foreign language audio-visual education program, 2004, (6): 27-32.

[4] Xie Jiacheng. Self-built English-Chinese parallel corpus and translation teaching [J]. Journal of Guangxi College of education, 2008, (1):73-76.

[5] Yu Jun, Wang Zhaohui. Construction and application of teaching oriented corpus based on comparative translation teaching method [J]. China translation, 2010, (5):57-62.

[6] Li Tuwang. Corpus and translation teaching [J]. China translation of science and technology, 2007 (3). 
[7] Xu Jiajin, Xu Zongrui. A study on the interaction of lexical chunks in Chinese college students' spoken English [J]. Foreign language teaching and research, 2007, (6): 437-443.

[8] Wang Kefei, Qin Hongwu, Wang Haixia. A preliminary study on the application of bilingual parallel corpus translation teaching platform. Foreign language audio-visual education program, 2007, (6): 3-8. 